\title{
Alimentation en Eau et Assainissement : rapport général (2ème partie)
}

\author{
Water supply and sanitation : general report 2
}

par Marie-Marguerite Bourbigot

Générale des Eaux

Producing sufficient quantities of drinking water is a great responsibility for the water industry, especially since the associated difficulties are growing alongside the ever-increasing levels of urbanisation across the world. The high investment levels required for water production and distribution projects, as well as for sewerage, show the need for internationally based cooperation and assistance. The French model rapidly became the accepted approach in countries where the Générale des Eaux and Suez Lyonnaise Groups are operating, and the international networks which have subsequently been developed with local partners have led to the sharing of ideas and acquired knowledge. These networks are increasingly developing around the major operators in the water industry. To ensure a rapid transfer of technology and knowledge, there is a need for a technical assistance network that can potentially be mobilised at any time and anywhere in the world. The adaptation of modelling software to new problems or new situations depends on the capacity of the users and the developers to group themselves around commercially available software. It is important to have a good balance between the increasingly rapid and high performance circuits interlinking the centres where technological development is concentrated and the training of staff able to industrialise and use these new systems. The ability to mobilise competences and place them at the service of field operators is one of the keys to successfully completing large projects. The examples presented by the new technical centre in Adelaide, the purification of the large sewerage collectors in Buenos Aires, the adaptation of modelling software to new problems using commercially available software, the consumption of chlorine within the system in Israel and the new challenges presented by standard procedures are all subjects treated in question 4 of the 25th Hydraulic Days and demonstrate that the full control of water, and ensuring its availability to rural and urban population, is task that is far from easy.

\section{I $\square$ INTRODUCTION}

L'eau est un facteur important pour le développement social et économique. Produire de l'eau potable et en quantité suffisante est une grande responsabilité pour l'industrie de l'eau. Mais les difficultés vont croissantes avec l'urbanisation galopante dans la majeure partie du monde. Les niveaux élevés d'investissements requis dans les projets de production et de distribution d'eau ainsi que d'assainissement montrent la nécessité d'une coopération et d'une assistance internationales.

\section{II $\square$ L'IMPLICATION DES ORGANISMES INTERNATIONAUX}

Cette assistance est de deux niveaux : financier et technique. Il convient de fournir des solutions économiques et fiables pour améliorer la qualité de l'eau à un coût acceptable en développant et adaptant les technologies aux contextes locaux. L'eau est un produit local et régional mais il existe un certain nombre de zones de recherche stratégiques où il est souhaitable que I'industrie de l'eau locale bénéficie d'une implication internationale.

Un exemple de coopération réussie est le centre technique créé à Adélaide (Australie Méridionale) par United Water International et ses compagnies mères, Générale des Eaux et Thames, et qui est représenté par Pascale Sztajnbok. United Water International qui a pris en charge la gestion et l'exploitation des usines de la région d'Adélaide s'est engagée à contribuer au développement économique local de l'Etat d'Australie Méridionale et à mettre en place une structure de Recherche et Développement. Cette cellule R\&D d'Adélaide peut se reposer sur le réseau international des compagnies mères Thames Water et Générale des Eaux. Il est, ainsi, souvent fait appel aux connaissances des experts de ces deux entités. Elle permet également une collaboration étroite avec les centres de recherche australiens dans leur domaine d'excellence qui ont souvent été développés autour des problèmes locaux (en Australie, par exemple : réutilisation des eaux usées, toxines algales). 


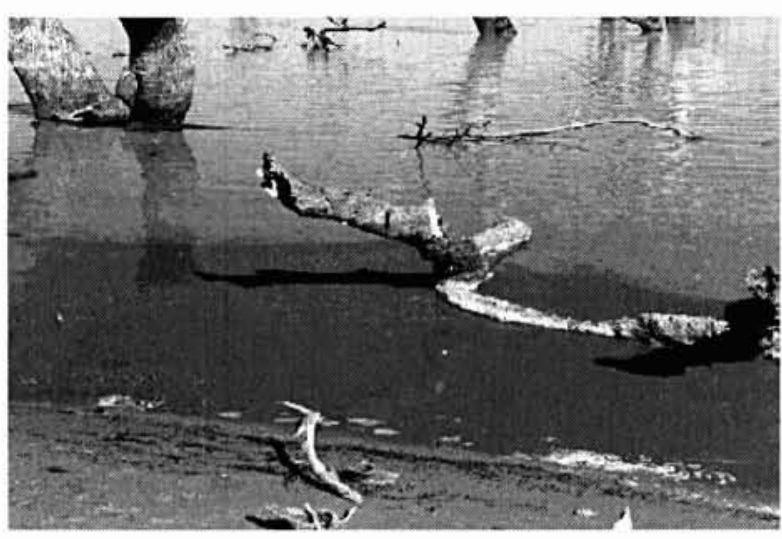

1. Toxines algales (paysage australien).

\section{III — UN FONCTIONNEMENT EN RÉSEAU ET PARTENARIATS}

Les deux grands groupes français ont eu des démarches assez proches dans la réorganisation de leur recherche. Ils ont, chacun, annoncé ces derniers mois la mise en réseau de leurs centres de recherche internationaux.

Les activités de recherche au sein du Groupe Générale des Eaux, en matière d'eau, se sont d'abord concrétisées par la création d'Anjou Recherche et d'un centre de recherche, à Maisons-Laffitte ; au sein du Groupe Suez Lyonnaise des Eaux par la création du CIRSEE au Pecq.

Ce modèle français a fait rapidement " école " dans les autres pays où les deux groupes se sont implantés, avec, pour Générale des Eaux, la création de structures de même type à Adélaide (Australie) avec United Water et près de Londres avec General Utilities (Angleterre). D'autres sont en cours de constitution en Asie (Kuala Lumpur et Hong Kong) et aux Etats-Unis (Metcalf and Eddy-PSG).

Les activités de recherche du Groupe Suez-Lyonnaise des Eaux sont réparties sur trois centres de recherche : CIRSEE (France), Northumbrian-Lyonnaise (Angleterre) et Astran (Kuala Lumpur).

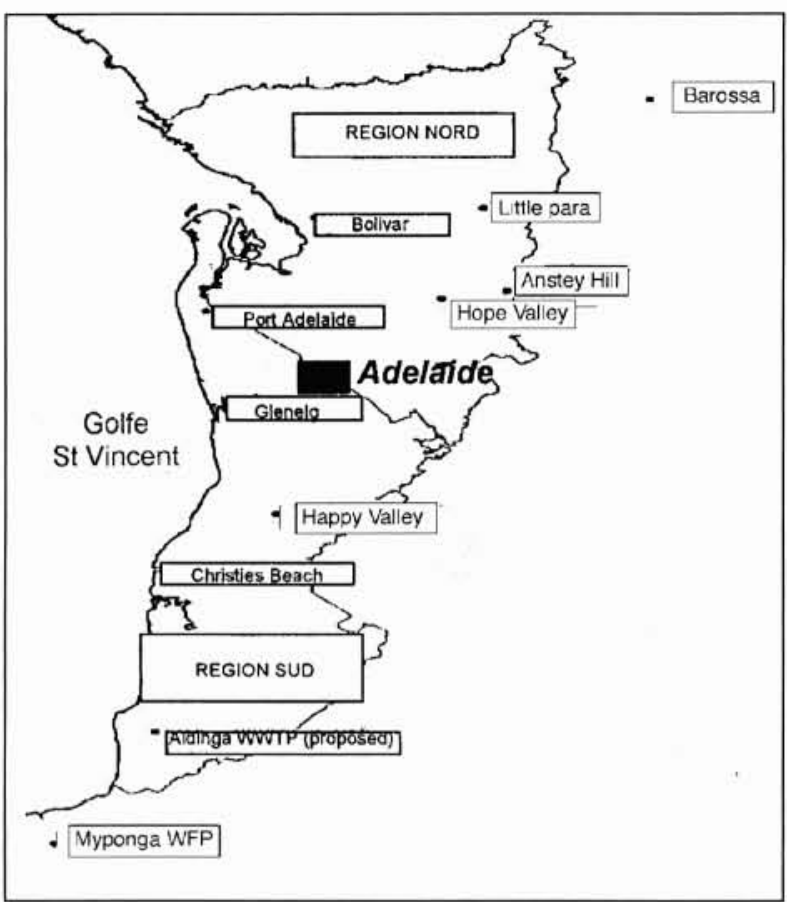

\section{Carte d'Australie Méridionale.}

Grâce à cette décentralisation, les groupes français profitent du contact avec d'autres organismes de recherche qui ont développé des compétences adaptées aux situations particulières rencontrées dans leur pays. Les deux groupes ne peuvent se contenter, pour gagner des marchés, de proposer des solutions uniquement conçues dans le cadre de l'“école française de l'eau".

Ces réseaux internationaux qui s'accroissent ainsi avec les partenariats locaux participent au partage des idées et des acquis. Les compétences sont développées à l'endroit où les besoins sont les plus immédiats. Un réseau de communication permet ensuite d'échanger des connaissances entre les différents experts techniques. De tels réseaux permettent d'accéder rapidement au bon moment aux données dont on a

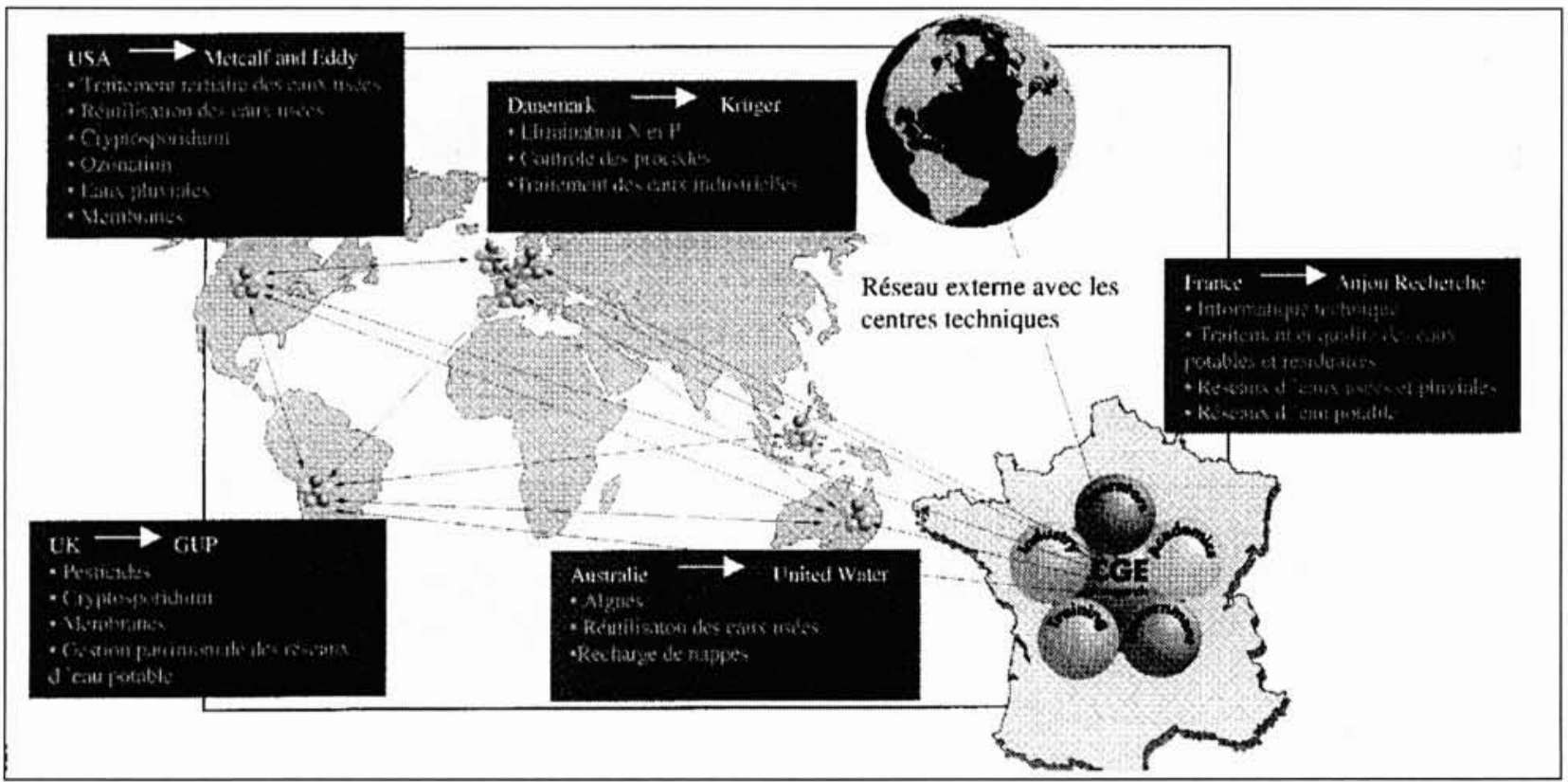

3. Réseaux des centres techniques de Générale des Eaux. 


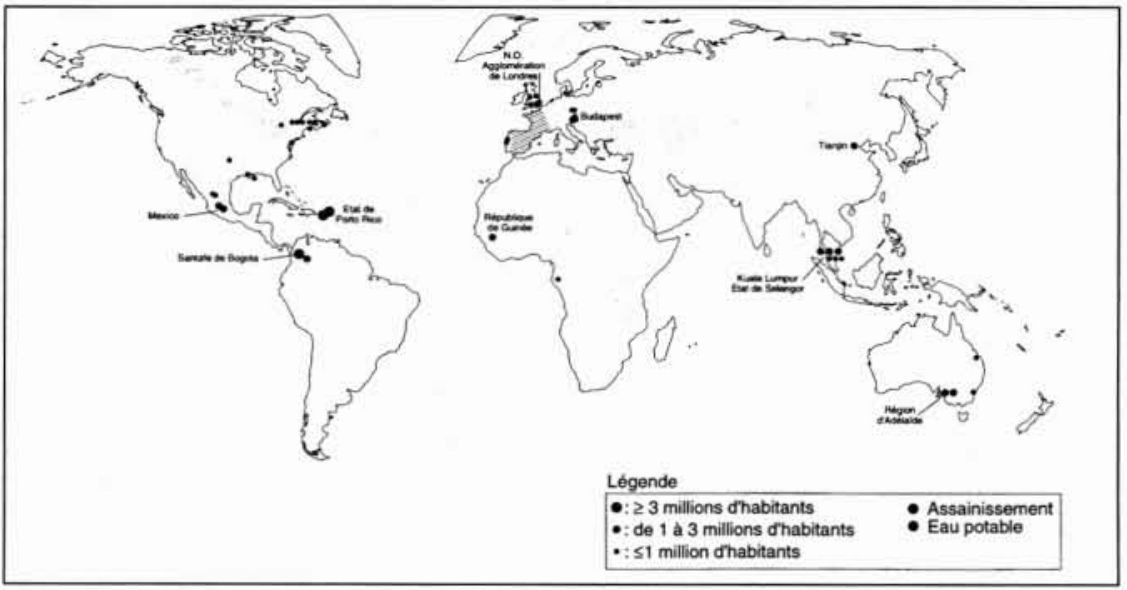

4. Principaux contrats de Générale des Eaux à l'étranger.

excellent exemple de partenariat réussi entre les ingénieurs de la Générale des Eaux et la société américaine Stoner Associates. Ce partenariat a permis de favoriser et d'accélérer le passage de la recherche à l'exploitation industrielle et d'obtenir un outil adapté à la configuration du réseau complexe de la banlieue de Paris $(8500 \mathrm{~km}$ de conduites, 45 usines de surpression, 51 réservoirs).

\section{D LES ENJEUX DE LA RECHERCHE}

La gestion active des infrastructures souterraines est une zone de recherche stratégique avec le développement des

réellement besoin dans l'action. Ils se développent de plus en plus autour des grands opérateurs de l'industrie de l'eau. Prenons le cas présenté par S. Doan Van pour le curage des grands collecteurs d'assainissement de Buenos Aires dont l'opérateur principal est le groupe Suez Lyonnaise. Le matériel a été mis au point dans un centre d'essais à Béziers sur un site reconstituant, à l'échelle 1 , la configuration des collecteurs de la capitale argentine.

Afin d'assurer un transfert rapide de la technologie et du savoir-faire, il faut pouvoir disposer d'un vivier potentiel d'assistance technique mobilisable à tout moment et dans le monde entier. Il ne suffit pas de développer une nouvelle technologie, il faut accélérer le passage de la recherche à l'exploitation industrielle, et former les équipes locales ainsi que le décrit S. Doan Van dans sa présentation du SONAR qui permet d'évaluer l'état du réseau de Buenos Aires. Ces études ont associé le personnel d'exploitation et se sont révélées des outils pédagogiques en formant les agents aux techniques d'auscultation et ont facilité le partage de l'expérience.

Laurent Phan souligne aussi l'importance de cette phase d'industrialisation et l'importance des partenariats à l'échelle mondiale. Il prend comme exemple, les outils de modélisation. D'outils de recherche, les modèles sont devenus des outils d'étude à l'usage des ingénieurs et des techniciens. Une certaine standardisation en fonction des besoins les plus couramment exprimés a donc eu lieu et pour un objectif d'étude bien défini, plusieurs modèles standardisés peuvent désormais se trouver en concurrence et être comparés. La tentation est grande de s'intéresser directement à ces outils intégrés d'aide à la gestion, mais ces outils ne sont pas toujours adaptables directement quand on est confronté à des conditions particulières, ce qui malheureusement arrive trop souvent. Il ne faut pas, pour cela, renoncer à ces outils mais savoir travailler en partenariat avec les développeurs de logiciels commerciaux. L'adaptation des logiciels de modélisation à de nouveaux problèmes ou de nouveaux cas de figures dépend de la capacité des utilisateurs et des développeurs à se regrouper autour des logiciels commerciaux pour explorer de nouvelles solutions et les faire valider sur le terrain. Ces partenariats s'établissent d'autant mieux que de grands opérateurs publics et privés ont leurs propres équipes de recherche et développement qui vont travailler étroitement avec la société commerciale.

Citons par exemple le développement du système de supervision centralisée du réseau de distribution d'eau de la banlieue parisienne présentée par Vincent Tiburce. C'est un outils de détection des fuites et d'évaluation des réseaux ou des modèles hydrauliques combinés avec un suivi de la qualité de l'eau, capables de surveiller les réseaux en temps réel. Nous avons un autre exemple avec la présentation de Y. Kadar qui décrit des travaux réalisés en Israël sur la consommation de chlore en réseau et la formation des sous-produits de chloration dans les réseaux de distribution longs et très maillés.

Les technologies de traitement des eaux sont considérées comme ayant atteint un certain niveau de maturité et les besoins en recherche additionnelle résideraient surtout dans l'optimisation des procédures mises en œuvre. Il faut être moins affirmatif car l'innovation est indissociablement liée au progrès. L'innovation et la qualité sont les meilleurs armes des entreprises. Et il y a encore de grands enjeux dans le traitement de l'eau et l'assainissement : le développement de procédés économiquement viables utilisant les technologies membranaires, la gestion et la valorisation des résidus de traitement, la réutilisation des eaux usées, la gestion des eaux pluviales et la gestion des infrastructures souterraines sont quelques exemples. Il y en a certainement d'autres.

Même dans les procédés qui sont considérés comme classiques comme la filtration sur sable, on peut rencontrer de nouveaux enjeux. P. Mazounie nous décrit tous les développements techniques qui ont accompagné la conception de l'usine de Prospect en Australie développée et exploitée par Australian Water Services, filiale de la Lyonnaise des Eaux. Les nouveaux filtres fonctionnent à une vitesse de filtration qui est le double de celle couramment appliquée sur des installations de filtration conventionnelles et se situent parmi les plus grands filtres jamais réalisés. Cela a permis une réduction significative du coût de la construction. D'autres développements techniques ont été réalisés dans le cadre de ce projet dans les domaines de l'hydraulique, du mélange des réactifs et de l'automatisation.

Tous ces exemples montrent que la maîtrise de l'eau, son accès par des populations rurales et urbaines, est une activité difficile.

Il est important d'avoir une bonne adéquation entre les circuits de plus en plus rapides et de plus en plus performants qui relient entre eux les pôles où se concentrent l'élaboration des technologies et la formation des hommes capables de les industrialiser et de les exploiter. La capacité à mobiliser les compétences et à les mettre au service des gens de " terrain" est une des clés de la réussite des grands projets. 